\title{
Influence of Different Methods Preparation on Platelet Activation in Stored Platelet Concentrates
}

\author{
Soleimany Ferizhandy Ali* \\ Iranian Blood Transfusion Research Center, High Institute for Research and Education in Transfusion Medicine, Tehran, Iran
}

\begin{abstract}
Background: Platelet concentrates are routinely manufactured from whole blood by differential centrifugation. During this storage period under blood bank conditions, biochemical, structural and functional changes occur, a process that is also known as platelet storage lesion. Their quality was assessed using the following parameters: platelets, leukocytes and erythrocytes counts, pH, CD63, lactate dehydrogenase and Annexin V.

Materials and Methods: In this experimental study, 25 platelet concentrates prepared with platelet rich plasma-platelet concentrates, 25 units via buffy coat and apheresis-derived platelet methods. The percentages of Annexin V, CD63 expression, lactatedehydrogenase, platelet, leukocytes counts and $\mathrm{pH}$ were evaluated.

Results: During storage for up to 5 days, no significant $\mathrm{pH}$, difference was observed among all three type of platelet concentrates $(p>0.05)$. The mean leukocytes count buffy coat units, platelet rich plasmaplatelet concentrates and apheresis-derived units were comparable and statistically significant difference was observed $(p<0.05)$. During storage for up to 5 days platelet concentrates units displayed significant an increase in lactatedehydrogenase, CD63 and Annexin V expressions, as compared with buffy coat units and apheresisderived units preparation on day $5(p<0.05)$.

Discussion: The kinetics of CD63 and annexin $V$ levels are influenced by the method used to prepare platelets for storage. The different levels of $C D 63$, annexin $\mathrm{V}$ and lactatedehydrogenase in three types of units clearly demonstrating a progressive activation process of platelet concentrates units exceeds that of buffy coat and apheresis-derived units. Further clinical studies will be necessary to determine whether meet the quality criteria or is superior in predicting in vivo viability.
\end{abstract}

Keywords: CD63; Annexin V; Buffy coat

\section{Introduction}

Blood banks optimize their protocols to yield the highest quality products in the shortest possible time. Optimized protocols may vary from blood bank to blood bank depending on which blood products are most desired. The issue of platelet quality during extended storage has been well addressed through studies using a variety of in vitro measures [1-3].

Platelet transfusion therapy has played an important role in the management of patients [4]. Today, a variety of methods for the preparation of platelets for transfusion are available, which may be due to different processing systems such as apheresis, buffy coat and platelet rich plasma platelet concentres methods [5,6]. Processing and storage may affect both platelet quality and function and they may thus be less effective when transfused than fresh platelets [7-9]. Platelet functional assessment can be performed by a number of different methods; including cell counting, $\mathrm{pH}$ and markers for platelet activation.

In the laboratory, phosphatidylserine exposure and its subsequent procoagulant activity can be assessed by several methods including flow cytometry utilising the binding of fluorescently labelled Annexin $\mathrm{V}$. The final stage of platelet activation is the capacity of the platelet to expose phosphatidylserine [10-12].

Annexin $\mathrm{V}$ was used as a parameter for quality monitoring of platelet concentrates during storage. Activation of platelet is followed by an increased CD63 expression; this protein secreted from the granules during storage [2,12-14]. Lactatedehydrogenase (LDH), a marker of disintegration of platelets as a parameter for activation marker of platelet concentrates during storage.
In summary this thesis has demonstrated assays to detect the quality in the platelet derived by different methods.

\section{Materials and Methods}

\section{Platelet preparation}

Preparation of buffy coat-platelet concentrate (BC): Twenty five whole blood was collected in a $450 \mathrm{ml}$ quadruple bag containing $63 \mathrm{ml}$ of CPDA1 anticoagulant (TERUMO PENPOL, Ltd., Puliyarakonam, Trivandrum, India). The whole blood was first subjected to "hard spin" centrifugation at $3640 \mathrm{rpm}$ for 7 minutes at $22^{\circ} \mathrm{C}$. After centrifugation the supernatant plasma and the subnanat red cells were transferred into attached satellite containers. Platelet poor supernatant was expressed into one satellite bag and buffy coat into another satellite bag. About $25-40 \mathrm{ml}$ of plasma was returned to buffy coat with the aim of cleaning the tubing from residual cells and obtaining an appropriate amount of plasma in the BC. The buffy coat was gently mixed with the plasma and again subjected to "light spin" centrifugation at $1180 \mathrm{rpm}$ for 8 minutes at $22^{\circ} \mathrm{C}$, along with one empty satellite bag. Briefly, the main difference lies in the amount of force applied in the first centrifugation step. In the

*Corresponding author: Soleimany Ferizhandy Ali, Iranian Blood Transfusion Research Center, High Institute for Research and Education in Transfusion Medicine, Tehran, Iran, Tel: 009888601559; E-mail: ferizhandy@yahoo.com

Received March 20, 2015; Accepted May 19, 2015; Published May 25, 2015

Citation: Ali SF (2015) Influence of Different Methods Preparation on Platelet Activation in Stored Platelet Concentrates. J Blood Disorders Transf 6: 279 doi:10.4172/2155-9864.1000279

Copyright: ( $) 2015$ Ali SF. This is an open-access article distributed under the terms of the Creative Commons Attribution License, which permits unrestricted use, distribution, and reproduction in any medium, provided the original author and source are credited. 
buffy-coat method, a higher gravitational $(\mathrm{g})$ force is applied causing the platelets and leukocytes to form a tight buffy-coat layer between the red cell and plasma layers suspended in the plasma.

Preparation of platelet rich plasma-platelet concentrates (PC): Four hundred and fifty $\mathrm{ml}$ of whole blood was collected in triple bag containing CPDA1 anticoagulant (JMS Singapore Pte Ltd) and PCs was prepared within 4 hours of collection stored for 5 days at $20-24^{\circ} \mathrm{C}$ under constant agitation $(n=25)$.

Aphaeresis-derived platelet concentrates (APC): Twenty five healthy volunteer donors each were subjected to this study by Haemonetics MCS plus cell separator. The apheresis sets used for the cell separator was REF 995E. This procedure was done in a closed system. During the procedures, the blood was anticoagulant at the point of withdrawal, and the ratio of whole blood and anticoagulant (ACD) was maintained at 9:1 to 11:1.

At the end of the collection procedure, the platelet collection bag was shaken vigorously to detach the platelets from the wall of the bag and kept for 1 hour at room temperature to make it an even suspension. The final volume of the APC ranged from $250-280 \mathrm{ml}$.

\section{Laboratory analysis}

On days $0,1,3,5$ samples from PC, BC and APC were taken for analysis under sterile condition. Manual cell counts were therefore carried out utilizing the improved Neubauer chamber for counting leukocytes (WBC) and platelets count. The $\mathrm{pH}$ was evaluated at the end of the day of storage. $\mathrm{PH}$ values were determined by the $\mathrm{pH}$ meter (Mettler Toledo, witzerland). LDH was determined using colorimetric methods by Roche Hitachi 902 Chemistry Analyzer (Roche, Germany). Using flow cytomerty, we investigated platelet membrane binding of Annexin V (Annexin V Fluorescein-FITC/ApoScreen)and CD63 (Mouse Monclonal Antibody to human CD63-FITC/Caltage) expression in platelet stored for up to 5 days under standard blood banking conditions. Special monoclonal antibodies that conjugated with fluorescence dye in flow cytometric method was used for its.

\section{Statistical analysis}

All data were expressed as mean $\pm \mathrm{SD}$. We performed statistical comparison by using ' $\mathrm{t}$ '-test. A probability of $\mathrm{P}<0.05$ (two-sided) was used to reject null hypothesis.

\section{Results}

In this experimental study, the mean platelet count of BCs, PCs and APCs was 5.7+1.9 $\times 10^{10} /$ unit, 5.8+2.1 $\times 10^{10} /$ unit and $2.4+2.2 \times 10^{11} /$ unit. The mean platelet count PCs, BCs and APCs was comparable and statistically significant difference was observed $(\mathrm{P}<0.05)$. The volume of individual units was calculated.The mean volume of the PCs, BCs and APCs was $55.6+12.3 \mathrm{ml}, 69+20.6 \mathrm{ml}$ and $245+15.6 \mathrm{ml}$. The mean WBC count in PC, BC and APCs units was $41+0.48 \times 10^{6} /$ unit, $23+0.49$ $\times 10^{6} /$ unit and $3.3+0.8 \times 10^{6} /$ unit. The mean leukocyte counts BCs, PCs and APCs was comparable and statistically significant difference was observed $(p<0.05)$. All units of platelet concentrates product met the recommended quality control parameters of volume. LDH and cell count. Apheresis product by Haemonetics MCS plus showed better leucoreduction than BCs and PCs product. Their mean $\mathrm{pH}$ was 6.9+0.21 (mean+SD) and ranged from 6.8-7.01 and no difference was observed between all three types of platelet concentrate. The results of $\mathrm{pH}$ and cell counts are listed in Table 1 .

The mean levels CD63 and percentage of Annexin V was comparable and statistically no significant difference was observed in three types of platelet concentrate on days $(0,1,3)$, but there was statistically relevant increase in CD63 and Annexin V on day 5. During storage for up to 5 days PCs units displayed a significant increase in the CD63, Annexin V expressions, as compared with BCs units and APCs units ( $\mathrm{p}<0.05)$. The mean LDH was comparable and statistically no significant difference was observed in three types of platelet concentrate on days $(0,1,3)$, but there was statistically increase in LDH on day 5 . The data showed that the level of LDH was significantly in the BCs units and APCs units than that PCs in the days 5 of storage $(\mathrm{P}$-value $<0.05)$. The platelet activation markers CD63, LDH and Annexin V are presented in Table 2.

\section{Discussion}

We found statistically significant differences in a variety of cellular, functional and flow cytometry parameters [15]. This study has determined in vitro quality of 5 days stored platelet concentrates prepared by three different methods. The detection of platelet activation has been used as a guide to the assessment of the suitability of techniques or conditions under which platelets have been prepared for transfusion. The present paper describes an in vitro comparison of different preparations. The studies suggested that the degree of in vitro activation as evidenced by the expression of CD63 and Annexin

\begin{tabular}{|c|c|c|c|c|c|c|c|c|c|c|c|c|}
\hline \multirow{2}{*}{ Day } & \multicolumn{4}{|c|}{ PRP-PC } & \multicolumn{4}{|c|}{ BC } & \multicolumn{4}{|c|}{ APC } \\
\hline & 0 & 1 & 3 & 5 & 0 & 1 & 3 & 5 & 0 & 1 & 3 & 5 \\
\hline $\begin{array}{l}\text { Platelet/unit } \\
\quad \times 10^{10}\end{array}$ & $57+1.9 \boldsymbol{\Lambda}$ & $5.7+2.1 \Delta$ & $5.9+1.8 \Delta$ & $5.4+2.4 \boldsymbol{\Delta}$ & $5.9+1 \boldsymbol{\Delta}$ & $5.9+1.2 \Delta$ & $5.6+2 \boldsymbol{\Delta}$ & $58+2.1 \Delta$ & $21+2 \Delta$ & $24+3.1 \boldsymbol{\Delta}$ & $25+2.1 \boldsymbol{\Delta}$ & $27+2 \boldsymbol{\Lambda}$ \\
\hline WBC/unit $\times 10^{6}$ & $44+0.65 \boldsymbol{\Delta}$ & $45+0.71 \boldsymbol{\Delta}$ & $42+0.78 \Delta$ & $42+1 \boldsymbol{\Delta}$ & $28+0.5 \Delta$ & $22+0.86 \Delta$ & $22+0.7 \Delta$ & $21+0.95 \boldsymbol{\Delta}$ & $3.3+0.65 \boldsymbol{\Delta}$ & $3.2+0.71 \boldsymbol{\Delta}$ & $3.1+0.78 \boldsymbol{\Delta}$ & $3.3+0.65 \boldsymbol{\Delta}$ \\
\hline $\mathrm{pH}$ & $6.9+0.12$ & $7+0.13$ & $7.01+0.29$ & $7.02+0.1$ & $6.6+0.12$ & $6.7+0.13$ & $7+0.2$ & $7+0.4$ & $6.9+0.12$ & $7+0.13$ & $7.01+0.29$ & $6.9+0.12$ \\
\hline
\end{tabular}

$\Delta$ Significant difference between BC,PC and APC; Values are mean+standard deviation; $\mathrm{n}=25$

Table 1: Platelet and white blood cells counts and $\mathrm{pH}$ in platelet rich plasma-platelet concentrates $\mathrm{PC}$ ), buffy coat-derived platelet concentrate (BC) and apheresis-derived platelet concentrate (APC)

\begin{tabular}{|c|c|c|c|c|c|c|c|c|c|c|c|c|}
\hline \multirow{2}{*}{ Day } & \multicolumn{4}{|c|}{ PRP-PC } & \multicolumn{4}{|c|}{$B C$} & \multicolumn{4}{|c|}{ APC } \\
\hline & 0 & 1 & 3 & 5 & 0 & 1 & 3 & 5 & 0 & 1 & 3 & 5 \\
\hline CD63\% & $13.4+9.1$ & $31+10.2$ & $46+14.7$ & $52+16 \Delta$ & $10.1+9.1$ & $27.3+9.4$ & $33.1+12$ & $40+13.1 \Delta$ & $9.9+8.7$ & $24.9+8.8$ & $30+10.4$ & $33+11.1 \boldsymbol{\Delta}$ \\
\hline Annexin V\% & $3.31+0.9$ & $6.3+2.1$ & $13.3+5.6$ & $19+6.4 \boldsymbol{\Delta}$ & $2.3+0.9$ & $4.8+1.6$ & $8.8+2.5$ & $11+4.5 \Delta$ & $2.1+0.8$ & $4.48+1.1$ & $7.5+2.6$ & $9.1+3.9 \boldsymbol{\Delta}$ \\
\hline $\begin{array}{l}\mathrm{LDH} \\
\mathrm{IU} / \mathrm{ml}\end{array}$ & $142+10$ & $149+12$ & $165+15$ & $223+12 \Delta$ & $129+9$ & $134+14$ & $142+12$ & $149+10 \Delta$ & $119+10$ & $121+10$ & $130+10$ & $139+12 \Delta$ \\
\hline
\end{tabular}

$\Delta$ Significant difference between BC, PC and APC; Values are mean+standard deviation; $\mathrm{n}=25$.

Table 2: $\mathrm{CD} 62 \mathrm{P} \mathrm{LDH}$, and Annexin $\mathrm{V} \%$ in platelet rich plasma-platelet concentrates (PC), buffy coat-derived platelet concentrate (BC) and apheresis-derived platelet concentrate (APC). 
Citation: Ali SF (2015) Influence of Different Methods Preparation on Platelet Activation in Stored Platelet Concentrates. J Blood Disorders Transf 6: 279. doi:10.4172/2155-9864.1000279

Page 3 of 3

$\mathrm{V}$ binding were dependent on the different preparative methods $[2,4,12,14]$.

We found that the extent of activation was significantly higher in PCs than in BCs and APCs units. It was concluded that, immediately after preparation, PCs are more activated than BCs and APCs units. This is most likely a result of the pelleting that follows the second highspeed centrifugation of the PCs.

However, the different CD63 and Annexin V in BCs and APCs clearly demonstrate that process of activation exceeds that of PCs and rises progressively during storage under blood bank conditions. Although in three preparations, platelet activation is increased by storage time, APCs are characterized by a much better in vitro than PCs $[3,5,7]$. However, the different CD63 and Annexin V in PCs and APCs clearly demonstrate that process of activation exceeds that of APCs. Platelets processed by the buffy coat technique showed less CD63 expression than platelets prepared by the PCs method. This study shows that in addition to $\mathrm{pH}$, volume and cell counts; in vitro platelet activation markers were used to monitor platelet $[3,13,14]$. These data suggest that measurement of CD63, Annexin V and LDH may be a more desirable markers for clinical studies of activated platelets, since it may be less susceptible to artifactual elevation due to minor variations in sample handling and assay $[3,12,14]$. The results of this study showed that the LDH levels were also increased during storage in platelet concentrates. Although LDH level was increased during the storage in the three methods, but the rise of LDH was significantly higher in PCs than that of Bc and APC methods. This study could imply the effects kind of methods for preparation on quality product. The production processes subject the platelets to different kinds of stresses and stimuli. The platelets produced by either apheresis or buffy-coat production methods show little evidence of activation than platelet concentrates (PCs) [16].

Although the ability of transfused platelet to circulate and function is deponent on both the effect of the ex-vivo storage lesions that undermines platelet functionality and the status of the in vivo milieu of the transfused individual.

\section{Acknowledgements}

Thanks to the Tehran blood service for providing platelet concentrates for this project. This project supported by Iranian blood transfusion organization-research centre.

\section{References}

1. Rinder HM, Murphy M, Mitchell JG, Stocks J, Ault KA, et al. (1991) Progressive platelet activation with storage: evidence for shortened survival of activated platelets after transfusion. Transfusion 31: 409-414.
2. Eolar G, Wihte JH (1995) Change in Glycoprotein expression after platelet activation. Thrombosis and Haemostasis 74: 352-357.

3. Rinder HM, Ault KA (1998) Platelet activation and its detection during the preparation of platelets for transfusion. Transfus Med Rev 12: 271-287.

4. Heaton WA, Rebulla P, Pappalettera M, Dzik WH (1997) A comparative analysis of different methods for routine blood component preparation. Transfus Med Rev 11: 116-129.

5. Fijnheer R, Pietersz RN, de Korte D, Gouwerok CW, Dekker WJ, et al. (1990) Platelet activation during preparation of platelet concentrates: a comparison of the platelet-rich plasma and the buffy coat methods. Transfusion 30: 634-638.

6. Böck M, Rahrig S, Kunz D, Lutze G, Heim MU (2002) Platelet concentrates derived from buffy coat and apheresis: biochemical and functional differences. Transfus Med 12: 317-324.

7. Jerad S, Prane K (1997) The Platelet Storage lesions. Transfusion Medicine Reviews 2: $130-144$.

8. Dijkstra-Tiekstra MJ, Pietersz RN, Huijgens PC (2004) Correlation between the extent of platelet activation in platelet concentrates and in vitro and in vivo parameters. Vox Sang 87: 257-263.

9. Matsubayashi H, Weidner J, Miraglia CC, Mclntyre JA (1999) Platelet membrane early activation markers during prolonged storage. Thromb Res 93 $151-160$.

10. Albanyan AM, Murphy MF, Rasmussen JT, Heegaard CW, Harrison P (2009) Measurement of phosphatidylserine exposure during storage of platelet concentrates using the novel probe lactadherin: a comparison study with annexin V. Transfusion 49: 99-107.

11. Tait JF, Smith C, Wood BL (1999) Measurement of phosphatidylserine exposure in leukocytes and platelets by whole-blood flow cytometry with annexin V. Blood Cells Mol Dis 25: 271-278

12. Hagberg IA, Lyberg T (2000) Blood platelet activation evaluated by flow cytometry: optimised methods for clinical studies. Platelets 11: 137-150.

13. Cardigan R, Williamson LM (2003) The quality of platelets after storage for 7 days. Transfus Med 13: 173-187.

14. Holme S, Sweeney JD, Sawyer S, Elfath MD (1997) The expression of p-selectin during collection, processing, and storage of platelet concentrates: relationship to loss of in vivo viability. Transfusion 37: 12-17.

15. Wang C, Mody M, Herst R, Sher G, Freedman J (1999) Flow cytometric analysis of platelet function in stored platelet concentrates. Transfus Sci 20: 129-139.

16. Kamath S, Blann AD, Lip GY (2001) Platelet activation: assessment and quantification. Eur Heart J 22: 1561-1571.

Citation: Ali SF (2015) Influence of Different Methods Preparation on Platelet 